\title{
LETTERS
}

\section{Hoarseness of unclear origin may be a sign of cerebrovascular disease and herald impending recurrent brainstem stroke}

I read with interest the piece by Morgan and Rigby regarding hoarseness of unclear origin in adults. ${ }^{1}$ The authors, from a division of otolaryngology, are rightly concerned about clinicians overlooking hoarseness as an early feature of laryngeal cancer, and they are to be commended for highlighting this subtle but sometimes ominous sign.

Although laryngeal cancer is indeed one worrisome cause of hoarseness, a newly hoarse voice can be ominous for other reasons too - one of which is ischemia in the brainstem. One common brainstem stroke syndrome, lateral medullary (Wallenberg) syndrome, involves the sudden onset of a constellation of neurologic deficits including hoarseness, ataxia, vertigo, dysphagia, ipsilateral Horner syndrome (miosis, partial ptosis, anhidrosis), hiccups, vomiting and ipsilateral facial numbness with crossed (contralateral) body numbness. Hoarseness occurs in $55 \%$ of patients with lateral medullary syndrome. ${ }^{2}$

However, when the infarct in the lateral medulla is small, the clinical syndrome is often incomplete. Therefore, it may be difficult for clinicians who are assessing hoarseness to spot the syndrome in its partial form. ${ }^{3}$ First, hoarse voice is the predominant presenting symptom of lateral medullary ischemia in some cases, with other more subtle signs evident only upon neurologic examination; 4,5 furthermore, hoarseness can persist chronically even after other symptoms have resolved. ${ }^{4}$ Second, when lateral medullary ischemia also causes dysphagia or vomiting, ${ }^{5,6}$ it can be easy to overlook hoarseness as the independent neurologic sign it sometimes is, and to inappropriately ascribe it to gastroesophageal reflux disease or aspiration.

Early recognitions of lateral medullary syndrome - like laryngeal cancer - is crucial, because any delay in diagnosis puts patients at risk for potentially fatal recurrent brainstem ischemia related to vertebral artery dissection or atheroembolism. For this reason, any patient presenting to their family physician with new hoarseness, whether chronic or sudden and acute, should undergo a neurologic examination with, at minimum, a search for pupillary asymmetry, nystagmus, finger-to-nose dysmetria or gait ataxia.

Although a referral to otolaryngology for laryngeal examination is certainly an important step in the diagnosis of iso- lated hoarseness of unclear origin in adults, clinicians must first be certain that the hoarseness is truly isolated to avoid introducing a dangerous delay in the management of what may be a neurologic emergency.

\section{J. Alexander Fraser MD}

Neurologist, Department of Clinical Neurological Sciences, Western University, London, Ont.

Cite as: CMAJ 2018 September 10; 190:E1088. doi: 10.1503/cmaj.70005

\section{References}

1. Morgan AP, Rigby MH. Hoarseness of unclear origin in adults. CMAJ 2018;190:E80.

2. Kim JS, Lee JH, Suh DC, et al. Spectrum of lateral medullary syndrome. Correlation between clinical findings and magnetic resonance imaging in 33 subjects. Stroke 1994;25:1405-10.

3. Ogawa K, Suzuki Y, Oishi M, et al. Clinical study of 46 patients with lateral medullary infarction. J Stroke Cerebrovasc Dis 2015;24:1065-74.

4. Baugh CW, Brown DF, Nadel ES. Horner's syndrome, hoarseness, and unsteady gait. J Emerg Med 2009;36:176-80.

5. Roldán-Valadez E, Juárez-Jiménez H, CoronaCedillo R, et al. Wallenberg syndrome: magnetic imaging findings and clinical correlation [article in Spanish]. Gac Med Mex 2007;143:429-32.

6. Castillo AL, Barahona-Garrido J, Criales S, et al. Wallenberg's syndrome: an unusual cause of dysphagia. Case Rep Gastroenterol 2007;1:135-43.

Competing interests: None declared. 